\title{
Large Disparity between Prevalence and Treatment Rates for Hepatitis C in Western China
}

\author{
Zhi-Wei Chen, Zhao Li, Qiao-He Wang, Xiao-Ling Wu, Hu Li, Hong Ren and Peng Hu* \\ Department of Infectious Diseases, Institute for Viral Hepatitis, The Key Laboratory of Molecular Biology for Infectious Diseases, \\ Chinese Ministry of Education, The Second Affiliated Hospital of Chongqing Medical University, Chongqing, China
}

\section{Abstract}

Background and Aims: Recently, the World Health Organization adopted the first-ever global hepatitis strategy with the dream of eliminating viral hepatitis as a public health threat by 2030. However, the epidemiology and treatment rates of hepatitis C virus (HCV) infection in Western China are still unknown. Methods: A total of 111,916 adult individuals (15-96 years) who underwent the HCV-antibody (HCV-Ab) test in the Second Affiliated Hospital of Chongqing Medical University between 2013 and 2015 were included in this study. We retrospectively analyzed the electronic medical records' data for each, and the positivity of HCV-Ab and the treatment of HCV RNA-positive patients were evaluated. Results: During 2013-2015, the crude prevalence of HCVAb was $1.4 \%(95 \% \mathrm{CI}: 1.4-1.5 ; 1,611 / 111,916)$ and the adjusted prevalence of HCV-Ab was $1.7 \%$ (95\%CI: $1.6-1.8$ ), which was higher than in the 2006 national study $(0.43 \%)$. The prevalence was 2-times higher in males than females (2.0\% vs. $1.1 \%, p<0.01)$. Notably, only $46 \%$ (434/951) of the HCV RNA-positive patients received standard peginterferon plus ribavirin treatment, with 370 (82\%) that completed treatment, of whom $272(74 \%)$ achieved sustained virologic response (SVR). Particularly, 11\% (32/292) of HCV RNA-positive patients were HBsAg-positive, and the SVR rate for them was lower than for the HBsAg-negative patients, but no significant difference was observed. Conclusions: HCV infection may have increased since 2006 in Western China. The SVR rate of peg-interferon plus ribavirin treatment was high, but the proportion of untreated HCV patients was large. Thus, more efforts need to be made by the government to create a scientific-based policy for HCV treatment and prevention.

Citation of this article: Chen ZW, Li Z, Wang QH, Wu XL, Li $\mathrm{H}$, Ren $\mathrm{H}$, et al. Large disparity between prevalence and treatment rates for hepatitis $C$ in Western China. J Clin Transl Hepatol 2018;6(4):385-390. doi: 10.14218/JCTH.2018.00027.

Keywords: Epidemiology; Hepatitis C virus; Hospital-based population study; Treatment.

Abbreviations: APRI, aspartate aminotransferase to platelet ratio index; CI, confidence interval; DAAs, direct-acting antiviral agents; HBV, hepatitis B virus; $H C V$, hepatitis $\mathrm{C}$ virus; $\mathrm{HBsAg}$, hepatitis $B$ surface antigen; $\mathrm{HCV}-\mathrm{Ab}, \mathrm{HCV}$ antibody; $\mathrm{P} / \mathrm{R}$ peg-interferon plus ribavirin; SD, standard deviation; SVR, sustained virologic response; WHO, World Health Organization.

Received: 11 April 2018; Revised: 30 July 2018; Accepted: 7 August 2018

* Correspondence to: Peng Hu, Department of Infectious Diseases, Institute for Viral Hepatitis, The Key Laboratory of Molecular Biology for Infectious Diseases, Chinese Ministry of Education, The Second Affiliated Hospital of Chongqing Medical University, 74 Linjiang Road, Yuzhong District, Chongqing 400010, China. Tel: +86-23-63693289, Fax: +86-23-63703790, E-mail: hp_cq@163.com

\section{Introduction}

Since its discovery in 1989, hepatitis C virus (HCV) infection has become a major public health issue worldwide and a leading cause of liver cirrhosis and liver cancer. ${ }^{1}$ According to estimates from the World Health Organization (WHO), 71 million people globally have chronic HCV infections, and approximately 399,000 people die each year from hepatitis C-related liver disease. ${ }^{2}$

Remarkably, the WHO recently adopted the first-ever global hepatitis strategy with the dream of eliminating viral hepatitis as a public health threat by 2030 , defined as a reduction in incidence by $90 \%$ and in mortality by $65 \% .^{2,3}$ To eliminate viral hepatitis, hepatitis $B$ and $C$ are the main obstacles. Unlike hepatitis B, the HCV infection is currently curable due to the direct-acting antiviral agents (DAAs) that are used as new short-course oral treatments for HCV infection. ${ }^{4}$ In this regard, it seems more feasible to achieve the goal with respect to HCV infection. However, a great deal of work is still needed, such as the prevention, screening and diagnosis of HCV, the accessibility of new drugs and the treatment of special populations, such as patients with hepatitis $B$ virus (HBV)/HCV coinfection. ${ }^{2,5-7}$

China has a heavy burden of viral hepatitis, ${ }^{1,8-10}$ making its situation severe. The HCV antibody (HCV-Ab) is a commonly available marker of HCV infection. ${ }^{9}$ However, the latest national population-based prevalence of $\mathrm{HCV}-\mathrm{Ab}$ in China was $0.43 \%$ in $2006 .{ }^{11}$ Hence, recent data on the prevalence of HCV-Ab, HCV RNA-positive and HBV/HCV coinfection in China are limited. Even though DAA treatment was approved in China recently, the high price limits the wide use of DAAs in China. Instead, the standard antiviral treatment regimen of $\mathrm{HCV}$ infection is peg-interferon plus ribavirin $(\mathrm{P} / \mathrm{R})$. At present, the proportion and outcomes for $\mathrm{HCV}$ patients who received $P / R$ treatment in the real world in China are still vague. To answer these questions, a hospitalbased population study seems more feasible and suitable.

This study retrospectively analyzed the epidemiology, diagnosis and treatment of $\mathrm{HCV}$ from a hospital-based population between 2013 and 2015, aiming to improve the prophylaxis and treatment of HCV infection in China to realize the magnificent target proposed by the WHO.

\section{Methods}

\section{Data collection}

This study was conducted by retrospectively analyzing electronic medical records from subjects presenting at the Second 
Affiliated Hospital of Chongqing Medical University between 1 January 2013 and 31 December 2015. Subjects were mainly from Western China (Chongqing Municipality, Sichuan and Guizhou Provinces).

A total of 111,916 adult subjects (aged $\geq 15$ years) who had HCV-Ab testing were included in this study. Data on patient demographics, dates of clinic visits, and HCV-Ab testing results were collected at each patient's first visit. When a patient's HCV-Ab was positive, data on HCV RNA testing and treatment during the study period were collected at the same time. On the other hand, hepatitis B surface antigen ( $\mathrm{HBsAg}$ ) testing was collected from HCV RNA-positive patients. And, markers for liver function and injury, including alanine transaminase, aspartate aminotransferase, gamma-glutamyltransferase, total bilirubin and direct bilirubin were collected from $\mathrm{HCV}$ RNA-positive patients. In addition, the aspartate aminotransferase to platelet ratio index (commonly known as APRI) was calculated to determine fibrosis in liver. The data collection for this study was approved by the Institutional Review Board of the Second Affiliated Hospital of Chongqing Medical University.

\section{Outcome variables}

The main outcome measures for this analysis were HCV-Ab and HCV RNA positivity, and the results of treatment. HCV-Ab positivity was defined as a positive result $(>1.0 \mathrm{~S} / \mathrm{co})$ by enzyme-linked immunosorbent assay. HCV RNA positivity was defined as detectable HCV RNA by real-time PCR. Sustained virologic response (SVR) was defined as undetectable HCV RNA obtained at 24 weeks after the end of treatment.

In order to have a better understanding of HCV diagnosis and treatment, two methods were used to examine the progression along the steps of the cascade of care: first, the percentage of subjects with HCV-Ab who completed each step, and second, the percentage of subjects at each step who moved to the next step.

\section{Statistical analysis}

Normally distributed variables were presented as mean \pm standard deviation (SD), and differences were tested by Student's $t$-test. Non-normally distributed variables are presented as median (interquartile range), and differences were assessed by Wilcoxon rank-sum test or Kruskal-Wallis test. Categorical variables are expressed as frequencies (\%), and differences were evaluated using the Pearson chi-square test. Crude prevalence estimations were weighted by poststratification for sex and age distribution in the population census of China in $2010 .{ }^{12}$ The $95 \%$ confidence intervals (95\%CIs) for crude and adjusted prevalence were computed using the Wilson score method and gamma distribution, respectively. A $p$-value $<0.05$ was considered statistically significant. The data were analyzed using the SPSS 17 software (SPSS Inc., Chicago, IL, USA).

\section{Results}

\section{Demographic characteristics}

A total of 111,916 individuals underwent HCV-Ab testing in this study between 1 January 2013 and 31 December 2015. The proportion of women was higher than men ( $41.6 \%$ male and $58.4 \%$ female). Most subjects represented the age group of
25-34 years-old (23.5\%); however, subjects aged $15-24$ yearsold only represented $6.5 \%$ of the overall subjects (Table 1 ).

\section{Crude and adjusted prevalence of $\mathrm{HCV}-\mathrm{Ab}$}

Overall and adjusted group-specific HCV-Ab prevalence in this study are listed in Table 1 . The total crude prevalence of HCV-Ab was $1.4 \%$ (95\%CI: 1.4-1.5). HCV-Ab positivity was observed more frequently in males than in females $(2.0 \% \mathrm{vs}$. $1.1 \%, p<0.01)$. The HCV-Ab prevalence presented a parabolic trend in age groups; that is, HCV-Ab positivity was the highest in the 35-44 age group (3.2\%, 95\%CI: $3.0-3.5$; $591 / 18,254)$ and gradually reduced on both sides with age (Table 1). Overall, individuals aged from 25-54 years represented $79.1 \%(1,274 / 1,611)$ of all HCV-Ab-positive subjects. To better estimate the prevalence of HCV-Ab, poststratification weight by population census of China in 2010 was conducted. The overall adjusted prevalence of HCV-Ab was $1.7 \%$ (95\%CI: $1.6-1.8)$. And, similar results of adjusted prevalence were observed in sex and age distributions (Table 1 ).

To investigate whether the prevalence of HCV-Ab had increased recently, we compared the prevalence of HCV-Ab with results from the latest national study in 2006. In general, the prevalence of HCV-Ab was higher in this 2013-2015 study than in the 2006 national study, regardless of the crude rate or adjusted rate (Fig. 1A). Further, in each age group, the prevalence was higher in men than in women and was higher in this 2013-2015 study than in the 2006 national study (Fig. 1B).

\section{Cascade of care for HCV-infected patients}

Among the 1,611 HCV-Ab-positive patients, 1,273 (79\%) had a confirmatory HCV-RNA test performed, of whom 947 (74\%) were found to be chronically infected (HCV RNA-positive). However, it is worth note that only $46 \%$ (434/947) of persons with chronic HCV infection initiated $\mathrm{P} / \mathrm{R}$ treatment, and 370 (82\%) completed treatment, of whom $74 \%$ (272/370) achieved SVR (Fig. 2).

In detail, of the $947 \mathrm{HCV}$ RNA-positive patients, the proportion receiving antiviral treatment in the $15-24$ age group was the highest $(63 \%, 12 / 19)$. Interestingly, a gradually declining trend in the SVR rate with increasing age was observed in this study. However, no difference was observed in either the HCV treatment or the SVR rate between males and females (Table 2).

\section{HBV/HCV coinfection in HCV RNA-positive patients}

Of the 947 HCV RNA-positive patients, 292 had testing results for HBsAg. The prevalence of HBsAg in patients with HCV was $11 \%(32 / 292 ;$ Fig. 3A). In addition, the SVR rate was lower in the HBsAg-positive patients than in the HBsAg-negative patients, but no significant differences were observed (Fig. 3B).

Further, to evaluate the effect of HBV on HCV, we screened the 292 patients' clinical data and eventually retrieved 122 patients' HCV RNA-level and data on liver function and injury, which included $14 \mathrm{HBV} / \mathrm{HCV}$ coinfected patients and $108 \mathrm{HCV}$ monoinfected patients. We found the HCV RNA level in the HBV/ HCV coinfected patients was lower than that in the HCV monoinfected patients, but the difference was not significant (6.0 vs. $6.1, p>0.05)$. In addition, there were no significant differences of alanine transaminase, aspartate aminotransferase, gammaglutamyltransferase, total bilirubin, direct bilirubin, platelet and APRI levels between the two groups (Table 3). 
Chen Z.W. et al: Low HCV treatment rates in Western China

Table 1. Demographic characteristics and prevalence of HCV-Ab in the hospital-based population study, 2013-2015

\begin{tabular}{lllll}
\hline & & & \multicolumn{2}{l}{ HCV-Ab-positive, \% $(95 \% \mathrm{CI})$} \\
\cline { 5 - 5 } Characteristic & Patients, $n(\%)$ & HCV-Ab-positive, $n$ & Crude prevalence & Adjusted prevalence \\
\hline $\begin{array}{l}\text { All } \\
\text { Sex }\end{array}$ & $111916(100.0)$ & 1611 & $1.4(1.4-1.5)$ & $1.7(1.6-1.8)$ \\
$\quad$ Male & $46609(41.6)$ & 916 & $2.0(1.8-2.1)$ & $2.3(2.1-2.4)$ \\
Female & $65307(58.4)$ & 695 & $1.1(1.0-1.1)$ & $1.1(1.0-1.2)$ \\
Age, y & & & & $0.7(0.5-0.9)$ \\
$15-24$ & $7300(6.5)$ & 42 & $0.6(0.4-0.8)$ & $1.7(1.6-1.9)$ \\
$25-34$ & $26305(23.5)$ & 323 & $1.2(1.1-1.4)$ & $3.4(3.1-3.6)$ \\
$35-44$ & $18254(16.3)$ & 591 & $3.2(3.0-3.5)$ & $2.0(1.8-2.2)$ \\
$45-54$ & $18306(16.4)$ & 360 & $2.0(1.8-2.2)$ & $0.8(0.7-0.9)$ \\
$55-64$ & $18925(16.9)$ & 149 & $0.8(0.7-0.9)$ & $0.6(0.5-0.7)$ \\
$\geq 65$ & $22826(20.4)$ & 146 & $0.6(0.5-0.7)$ & $<0.01$ \\
\hline
\end{tabular}

Abbreviations: Ab, antibody; CI, confidence interval; $\mathrm{HCV}$, hepatitis C virus.

\section{Discussion}

Hepatitis $\mathrm{C}$ has been the fourth most commonly reported infectious disease in China. ${ }^{13}$ In this hospital-based study of 111,916 individuals in Western China, the overall crude and adjusted prevalence of $\mathrm{HCV}-\mathrm{Ab}$ was $1.4 \%$ (95\%CI: $1.4-1.5$ ) and $1.7 \%(95 \% \mathrm{CI}: 1.6-1.8)$, respectively. This regional prevalence (found in 2013-2015) is lower than that found by national surveys of the whole population in $1992(3.2 \%)^{14}$ but higher than the whole population from $2006(0.43 \%)$ and in the Western China population in $2006(0.40 \%) .{ }^{11}$ In addition, recent studies ${ }^{13,15,16}$ from other places in China also reported a higher prevalence of HCV-Ab than that observed in 2006. All of the evidence has indicated that the HCV-Ab prevalence may have increased in China to some extent in recent years.

Interestingly, $\mathrm{HCV}$ infection was observed more often in males than females in this study. In fact, males showed nearly double the HCV infection rate than females, which is different from other studies that have reported equality between sexes. ${ }^{11,17-20}$ Several reasons may account for this discrepancy.
First, women were more likely to clear the virus spontaneously. Second, the geographical and racial differences should be considered. Finally, an increased risk for men to be infected by HCV may be due to unhealthy lifestyles or behaviors, such as unhealthy sexual activities, drug abuse, smoking and drinking.

In addition, $\mathrm{HCV}-\mathrm{Ab}$ positivity was mainly observed in the 25-54 age group (79\% of the total HCV-Ab-positive subjects). This is similar to the results from the study by Zhao et al., ${ }^{15}$ who reported a high HCV-Ab-positive rate in the 40-59 age group in the Hebei Province in China. The illegal blood trade of the 1990s in China may be an indispensable reason. Another explanation is the sporadic transmission of virus persisting in the community/village through the years. Moreover, an increase in mortality among chronic hepatitis $C$ patients may explain the decreased prevalence of HCV-Ab in older age groups.

According to guidelines from the $\mathrm{WHO}^{5}$ all adults and children with chronic HCV infection should be assessed for antiviral treatment. However, in this study, fewer than half of the
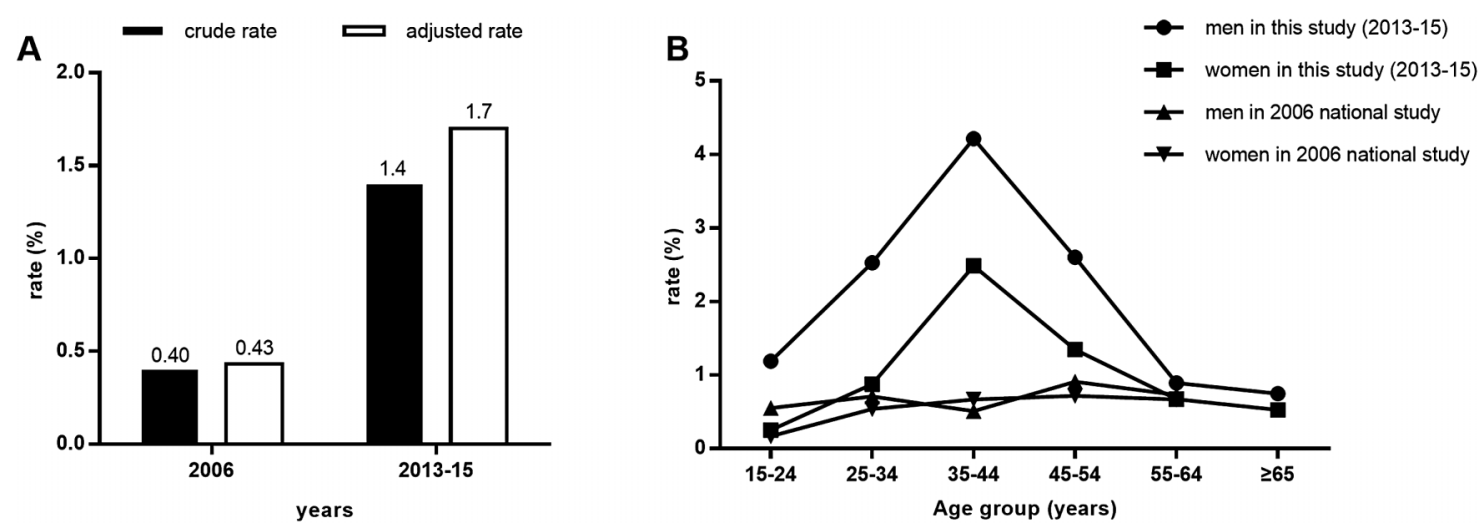

Fig. 1. Overall prevalence (A) and age-specific prevalence of HCV-Ab stratified by sex (B) in this $2013-15$ study and the 2006 national study*.

* Prevalence of HCV-Ab of the age 55-64 group in 2006 national study was used for data from the patients age 55-59 group because the data of the age $\geq 60$ group were unavailable in the national study.

Abbreviations: Ab, antibody; HCV, hepatitis $\mathrm{C}$ virus. 


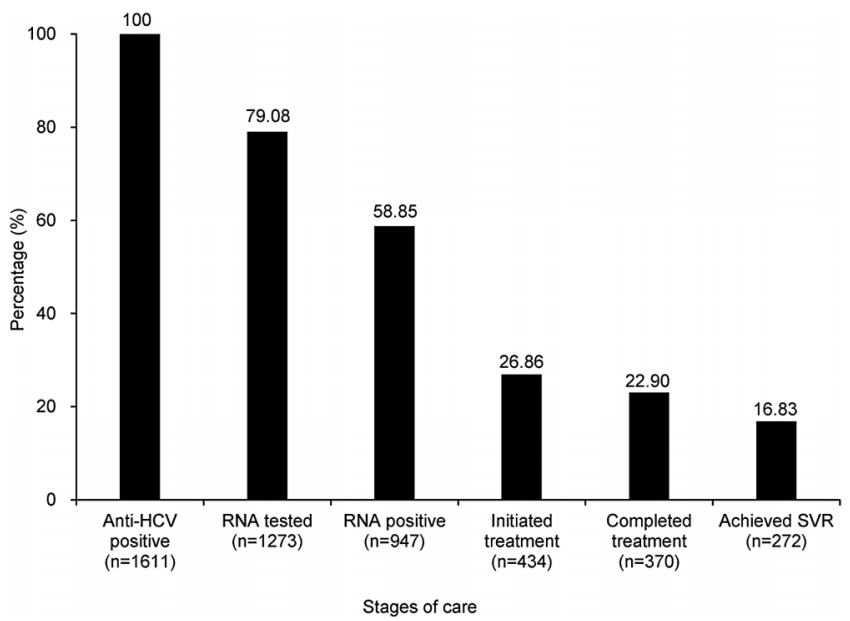

Fig. 2. Percentages for 1611 HCV-Ab-positive patients, showing cascade of care.

Abbreviations: Ab, antibody; HCV, hepatitis C virus; SVR, sustained virologic response.

patients received a standard antiviral treatment. This is not far enough. There are some reasons for this, such as publicity and education, drug price and medical insurance, but there needs to be more attention from the nation health policymakers. In this study, the highest proportion of those receiving $\mathrm{P} / \mathrm{R}$ treatment was still observed in the 15-24 age group $(63 \%)$, which is consistent with a study performed by Young et al. ${ }^{21}$ In addition, DAAs have not yet reached widespread use in China. Therefore, the standard antiviral treatment for $\mathrm{HCV}$ is still $P / R$ treatment. In this study, the SVR rate of $P / R$ treatment for $\mathrm{HCV}$ was $74 \%$. This is similar to the results of some recent studies. ${ }^{22-24}$ Interestingly, this study also found that the SVR rate of $P / R$ treatment was inversely related to increasing age. Wu et al. ${ }^{23}$ and Sezaki et al. ${ }^{25}$ also observed a similar phenomenon. Both of their results showed that older

Table 2. $P / R$ treatment of HCV infection and SVR rate in the hospitalbased population study, 2013-2015

\begin{tabular}{lllllll}
\hline & \multicolumn{2}{l}{ HCV treatment } & & \multicolumn{2}{l}{ SVR rate } & \\
\cline { 2 - 3 } Characteristic & $\mathrm{n} / \mathrm{N}$ & $\%$ & & $\mathrm{n} / \mathrm{N}$ & $\%$ & $p$-value \\
\hline All & $434 / 947$ & 46 & & $272 / 370$ & 74 & \\
Sex & & & & & $>0.05$ \\
$\quad$ Male & $203 / 461$ & 44 & & $120 / 172$ & 70 & \\
$\quad$ Female & $231 / 486$ & 48 & & $152 / 198$ & 77 & \\
Age, y & & & & & $<0.01$ \\
$15-24$ & $12 / 19$ & 63 & $8 / 9$ & 89 & \\
$25-34$ & $98 / 195$ & 50 & $74 / 88$ & 84 & \\
$35-44$ & $166 / 335$ & 50 & $103 / 138$ & 75 & \\
$45-54$ & $96 / 231$ & 42 & $58 / 84$ & 69 & \\
$55-64$ & $47 / 100$ & 47 & $21 / 37$ & 57 & \\
$\geq 65$ & $15 / 67$ & 22 & $8 / 14$ & 57 & \\
\hline
\end{tabular}

Abbreviations: $\mathrm{HCV}$, hepatitis $\mathrm{C}$ virus; $\mathrm{P} / \mathrm{R}$, pegylated-interferon plus ribavirin; SVR, sustained virologic response.
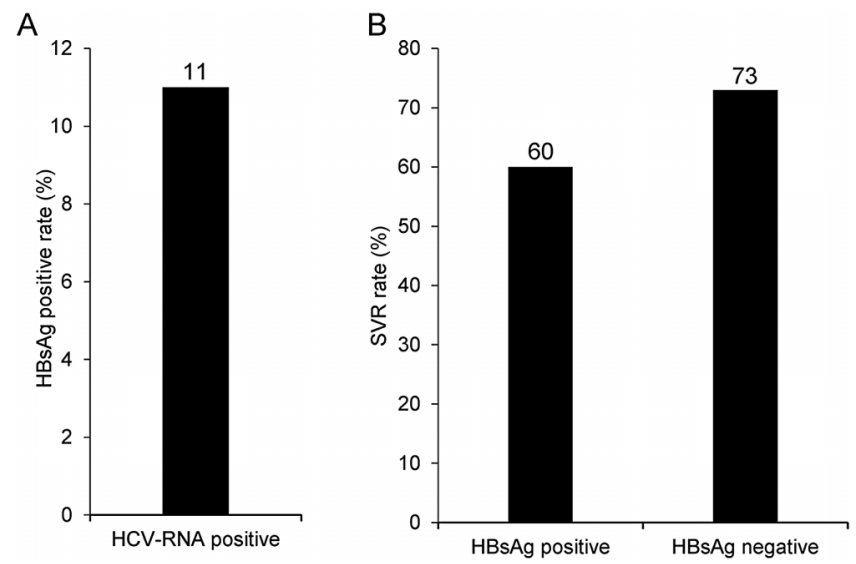

Fig. 3. Prevalence of HBsAg-positive (A) and the SVR rate of $P / R$ treatment between HBsAg-positive and -negative groups (B) in HCV-infected patients.

Abbreviations: HBsAg, hepatitis B surface antigen; HCV, hepatitis C virus; P/R, pegylated-interferon plus ribavirin; SVR, sustained virologic response.

patients had a lower tendency to achieve an antivirus response.

In addition, patients with HBV/HCV coinfection are still a special population, with studies showing interactions between these two viruses. It is not yet clear whether HBV and HCV interfere with each other's life cycles and reduce the effects of antiviral treatment. Notably, very recently, the U.S. Food \& Drug Administration has issued a black box warning for HCV drugs, due to its risk of reactivating hepatitis $\mathrm{B} .{ }^{26}$ So, it has become more important for us to know how many of the HCV patients have HBV/HCV coinfection. In this study, 11\% (32/292) of HCV RNA-positive patients also had HBV infection. This is much more than the rate observed in the USA $(1.3-5.8 \%)^{27-29}$ and in China $(4.11 \%)$ previously. ${ }^{30}$ The different region and sample size may be the reasons for this discrepancy. Moreover, as expected, the SVR rate of HBV/HCV coinfection was lower than HCV monoinfection in this study, but no statistically significant difference was observed $(60 \%$ vs. $73 \%, p>0.05)$. The number of patients who had a HBV marker test in this study was relatively few, and further large-scale study is needed in the future. Additionally, our results showed that HBV has little effect on HCV, which was consistent with previous report. ${ }^{28}$

There are several advantages in this study. First, this study was conducted with a large sample size $(111,916$ participants). Second, this study was novel and analyzed the prevalence of HCV-Ab over the last 3 years. Third, when analyzing the treatment of HCV RNA-positive patients, a hospital-based population was more feasible and suitable. However, limitations should also be considered in this study. First, when analyzing the prevalence of HCV-Ab, choosing hospital-based subjects as a target group was a challenge because this sub-population could not be completely equivalent to the general population. The hospital population included health candidates undergoing routine annual medical examination and patients from the department of infectious disease. To minimize the population bias, we excluded the patients from the department of infectious disease and found the prevalence of HCV-Ab to be $0.64 \%$, which was still higher than the national HCV-ab-positive rate 
Chen Z.W. et al: Low HCV treatment rates in Western China

Table 3. Demographic and clinical characteristics between HBV/HCV coinfected and HCV monoinfected patients

\begin{tabular}{llll}
\hline Characteristic & HBV/HCV, $n=14$ & HCV, $n=108$ & $p$-value \\
\hline Age in $y$, mean \pm SD & $51.0 \pm 12.7$ & $46.5 \pm 13.1$ & 0.229 \\
Male, $n(\%)$ & $7(50.0)$ & $58(53.7)$ & 0.794 \\
HCV-RNA as $\log _{10} \mathrm{IU} / \mathrm{mL}$, mean \pm SD & $6.0 \pm 1.3$ & $6.1 \pm 1.1$ & 0.814 \\
ALT as U/L, IQR & $85(41-114)$ & $65(35-141)$ & 0.740 \\
AST as U/L, IQR & $65(40-143)$ & $60(36-115)$ & 0.865 \\
GGT as U/L, IQR & $64.5(34-119)$ & $53(26-149)$ & 0.701 \\
TB as $\mu \mathrm{mol} / \mathrm{L}$, IQR & $17.5(9.8-27.3)$ & $15(10.0-20.8)$ \\
DB as $\mu \mathrm{mol} / \mathrm{L}$, IQR & $8(5.0-13.3)$ & $6(5-10)$ & 0.685 \\
PLT as $10 \wedge 9 / L$, mean $\pm S D$ & $122.1 \pm 65.4$ & $148.0 \pm 71.5$ \\
APRI level, IQR & $1.5(0.6-4.9)$ & $1.4(0.6-3.3)$ \\
\hline
\end{tabular}

Abbreviations: ALT, alanine transaminase; APRI, aspartate aminotransferase to platelet ratio index; AST, aspartate aminotransferase; DB, direct bilirubin; GGT, gammaglutamyltransferase; HBV, hepatitis B virus; HCV, hepatitis C virus; IQR, interquartile range; PLT, platelet; SD, standard deviation; TB, total bilirubin.

in 2006. Second, the data for HCV genotypes were not acquired in this study. HCV genotype testing requires a full set of equipment and complicated techniques, which had not been established in our hospital during the study period. Third, we did not collect information on the potential exposure to HCV infection, such as history of intravenous drug abuse, blood donation and family history of HCV infection. Most of this information in the electronic medical records of each patient was incomplete, which hindered further analysis. Despite these disadvantages, to our knowledge, this study revealed a high SVR rate and low treatment rate of chronic hepatitis $C$ patients in a hospital-based population in Western China for the first time.

In conclusion, this study reveals that HCV infection may have increased since 2006 in Western China. The SVR rate of $\mathrm{P} / \mathrm{R}$ treatment for HCV infection was high, but there is still a huge gap between higher prevalence and lower treatment of $\mathrm{HCV}$ infection. Hence, more efforts must be made by the government to create policy for HCV treatment and prevention scientifically, so we can achieve the magnificent goal proposed by the WHO.

\section{Acknowledgments}

This work was supported in part by the National Natural Science Foundation of China (30930082, 81171561 and 30972584) and the National Science and Technology Major Project of China (2008ZX10002-006, 2012ZX10002007001, 2017ZX10202203-007 and 2017ZX10202203-008).

\section{Conflict of interest}

The authors have no conflict of interests related to this publication.

\section{Author contributions}

Conception of study design (HR, PH), data collection (ZWC, $\mathrm{ZL}, \mathrm{XLW}, \mathrm{QHW}$ ), data analysis (ZWC, ZL), statistical analysis $(\mathrm{HL})$ and drafting the article (ZWC). All authors have approved the final version of the manuscript.

\section{References}

[1] Shepard CW, Finelli L, Alter MJ. Global epidemiology of hepatitis C virus infection. Lancet Infect Dis 2005;5:558-567. doi: 10.1016/S1473-3099(05) 70216-4.

[2] World Health Organization. Hepatitis C. Available from: http://www.who. int/en/news-room/fact-sheets/detail/hepatitis-c, accessed June, 2018.

[3] Wiktor SZ, Hutin YJ. The global burden of viral hepatitis: better estimates to guide hepatitis elimination efforts. Lancet 2016;388:1030-1031. doi: 10. 1016/S0140-6736(16)31018-2.

[4] Adinolfi LE, Guerrera B. All-oral interferon-free treatments: The end of hepatitis $C$ virus story, the dream and the reality. World J Hepatol 2015;7:23632368. doi: 10.4254/wjh.v7.i22.2363.

[5] World Health Organization. Guidelines for the screening, care and treatment of persons with chronic hepatitis C infection: Updated version, April 2016. Available from: http://www.who.int/hepatitis/publications/hepatitis-cguidelines-2016/en.

[6] Bruggmann P, Grebely J. Prevention, treatment and care of hepatitis C virus infection among people who inject drugs. Int J Drug Policy 2015;26:S22S26. doi: 10.1016/j.drugpo.2014.08.014.

[7] Stanaway JD, Flaxman AD, Naghavi M, Fitzmaurice C, Vos T, Abubakar I, et al. The global burden of viral hepatitis from 1990 to 2013: findings from the Global Burden of Disease Study 2013. Lancet 2016;388:1081-1088. doi: $10.1016 /$ S0140-6736(16)30579-7

[8] WHO Technical Consultation on a comprehensive National Hepatitis Programme in China with a focus on viral hepatitis B. and C treatment Meeting report. Availabe from: http://www.cfhpc.org/Downloads/who2014.pdf, accessed February 2014

[9] Mohd Hanafiah K, Groeger J, Flaxman AD, Wiersma ST. Global epidemiology of hepatitis $C$ virus infection: new estimates of age-specific antibody to HCV seroprevalence. Hepatology 2013;57:1333-1342. doi: 10.1002/hep.26141.

[10] Wang FS, Fan JG, Zhang Z, Gao B, Wang HY. The global burden of liver disease: the major impact of China. Hepatology 2014;60:2099-2108. doi: 10.1002/hep.27406.

[11] Chen YS, Li L, Cui FQ, Xing WG, Wang L, Jia ZY, et al. A sero-epidemiological study on hepatitis C in China. Zhonghua Liu Xing Bing Xue Za Zhi 2011;32: 888-891.

[12] Tabulation on the 2010 population census of the People Republic of China. Available from: http://www.stats.gov.cn/tjsj/pcsj/rkpc/6rp/indexch.htm.

[13] Zhou M, Li H, Ji Y, Ma Y, Hou F, Yuan P. Hepatitis C virus infection in the general population: A large community-based study in Mianyang, West China. Biosci Trends 2015;9:97-103. doi: 10.5582/bst.2015.01033.

[14] Xia GL, Liu CB, Cao HL, Bi SL, Zhan MY, Su CA, et al. Prevalence of hepatitis B and $C$ virus infections in the general Chinese population. Results from a nationwide cross-sectional seroepidemiologic study of hepatitis A, B, C, D, and $E$ virus infections in China, 1992. International Hepatology Communications 1996;5:62-73. doi: 10.1016/S0928-4346(96)82012-3.

[15] Zhao Y, Shen L, Ma J, Gao Z, Han X, Qi S, et al. Epidemiology of hepatitis C virus infection and risk factor analysis in the Hebei Province, China. PLoS One 2013;8:e75586. doi: 10.1371/journal.pone.0075586.

[16] Zhang Q, Qi W, Wang X, Zhang Y, Xu Y, Qin S, et al. Epidemiology of hepatitis $B$ and hepatitis $C$ infections and benefits of programs for hepatitis prevention in Northeastern China: A cross-sectional study. Clin Infect Dis 2016;62: 305-312. doi: 10.1093/cid/civ859. 
[17] Lu J, Zhou $Y$, Lin $X$, Jiang $Y$, Tian $R$, Zhang $Y$, et al. General epidemiological parameters of viral hepatitis $A, B, C$, and $E$ in six regions of China: a crosssectional study in 2007. PLoS One 2009;4:e8467. doi: 10.1371/journal. pone.0008467.

[18] Kim DY, Kim IH, Jeong SH, Cho YK, Lee JH, Jin YJ, et al. A nationwide seroepidemiology of hepatitis $C$ virus infection in South Korea. Liver Int 2013;33: 586-594. doi: 10.1111/liv.12108.

[19] Liu F, Chen K, He Z, Ning T, Pan Y, Cai H, et al. Hepatitis C seroprevalence and associated risk factors, Anyang, China. Emerg Infect Dis 2009;15:18191822. doi: 10.3201/eid1511.090263.

[20] Uhanova J, Tate RB, Tataryn DJ, Minuk GY. A population-based study of the epidemiology of hepatitis $C$ in a North American population. J Hepatol 2012; 57:736-742. doi: 10.1016/j.jhep.2012.05.018.

[21] Young KL, Huang W, Horsburgh CR, Linas BP, Assoumou SA. Eighteen- to 30year-olds more likely to link to hepatitis $C$ virus care: an opportunity to decrease transmission. J Viral Hepat 2016;23:274-281. doi: 10.1111/jvh.12489.

[22] Nguyen LH, Nguyen MH. Systematic review: Asian patients with chronic hepatitis C infection. Aliment Pharmacol Ther 2013;37:921-936. doi: 10.1111/apt. 12300.

[23] Wu Q, Zhan FY, Chen EQ, Wang C, Li ZZ, Lei XZ. Predictors of pegylated interferon alpha and ribavirin efficacy and long-term assessment of relapse in patients with chronic hepatitis $\mathrm{C}: \mathrm{A}$ one-center experience from China. Hepat Mon 2015;15:e28836. doi: 10.5812/hepatmon.15(6)2015.28836.

[24] Yu JW, Wang GQ, Sun L], Li XG, Li SC. Predictive value of rapid virological response and early virological response on sustained virological response in HCV patients treated with pegylated interferon alpha-2a and ribavirin. J Gastroenterol Hepatol 2007;22:832-836. doi: 10.1111/j.1440-1746.2007.04904.X.

[25] Sezaki H, Suzuki F, Kawamura Y, Yatsuji H, Hosaka T, Akuta N, et al. Poor response to pegylated interferon and ribavirin in older women infected with hepatitis C virus of genotype $1 \mathrm{~b}$ in high viral loads. Dig Dis Sci 2009;54: 1317-1324. doi: 10.1007/s10620-008-0500-y.

[26] FDA Drug Safety Communication: FDA warns about the risk of hepatitis B reactivating in some patients treated with direct-acting antivirals for hepatitis C. Available from: https://www.fda.gov/Drugs/DrugSafety/ucm522932. htm, accessed November 2016.

[27] Caccamo G, Saffioti F, Raimondo G. Hepatitis B virus and hepatitis C virus dual infection. World J Gastroenterol 2014;20:14559-14567. doi: 10. 3748/wjg.v20.i40.14559.

[28] Tyson GL, Kramer JR, Duan Z, Davila JA, Richardson PA, El-Serag HB. Prevalence and predictors of hepatitis B virus coinfection in a United States cohort of hepatitis C virus-infected patients. Hepatology 2013;58:538-545. doi: 10.1002/hep. 26400 .

[29] Bini EJ, Perumalswami PV. Hepatitis B virus infection among American patients with chronic hepatitis $C$ virus infection: prevalence, racial/ethnic differences, and viral interactions. Hepatology 2010;51:759-766. doi: 10. 1002/hep.23461.

[30] Yan LB, Rao HY, Ma Y], Bai L, Chen EQ, Du LY, et al. Hepatitis B virus infection in Chinese patients with hepatitis $C$ virus infection: prevalence, clinical characteristics, viral interactions and host genotypes: a nationwide cross-sectional study. BMJ Open 2016;6:e012016. doi: 10.1136/bmjopen-2016-012016. 